\title{
and jaw osteonecrosis: a case report
}

\begin{abstract}
Affiliations:
${ }^{1}$ Service de Stomatologie et Chirurgie Maxillo-Faciale, Cliniques Universitaires Saint-Luc, Université Catholique de Louvain, Av.

Hippocrate 10, 1200 Bruxelles, Belgique

Corresponding author: Magremanne M, Service de Stomatologie et Chirurgie Maxillo-Faciale, Cliniques Universitaires Saint-Luc, Université Catholique de Louvain, Av. Hippocrate 10, 1200 Bruxelles, Belgique

Email: michele.magremanne@uclouvain.be

ORCID ID: 0000-0002-5476-9355

Disclaimer: the views expressed in the submitted article are our own and not an official position of the institution or funder.
\end{abstract}


Dear Editor-in-Chief,

Please receive our article titled "Paget's disease of bone biphosphonates and jaw osteonecrosis: a case report" for open evaluation in Nemesis journal.

1) Summarize the study's contribution to the scientific literature: Bisphosphonates have been the treatment of choice of Paget's disease since the 1990s. Medication related osteonecrosis of the jaw (MRONJ) is a rare event in non oncologic patients. We describe a rare case of Paget's disease involving the maxilla with osteonecrosis in a context of bisphosphonate treatment.

2) Relate the study to previously published work: Maxillofacial involvement of Paget's disease occurs in less than $15 \%$ of cases. There is a lack of information in the literature about the association of MRONJ and Paget's disease.

3) Specify the type of article (for example, research article, systematic review, meta-analysis, clinical trial): we provide with a case report.

4) Describe any prior interactions with Nemesis regarding the submitted manuscript: we have no prior interactions with Nemesis journal.

5) Nemesis aim and scope relevance: side effect of bisphosphonate treatment 
Abstract

Objective: Paget's disease of bone is characterized by a focal increase in bone resorption and accelerated bone formation leading to a weaker and disorganised bone. Bisphosphonates (BPs) have been the treatment of choice of Paget's disease since the 1990s. Medication related osteonecrosis of the jaw (MRONJ) is a rare event in non oncologic patients. We describe a rare case of Paget's disease involving the maxilla with osteonecrosis in a context of bisphosphonate treatment.

Case report: an 87-year-old woman presented with 4 episodes of bone necrosis in 15 years. In this case report there is a clear chronologic association between the occurrence of MRONJ and the administration of iv BP for Paget's disease.

Maxillofacial involvement of Paget's disease occurs in less than $15 \%$ of cases. There is a lack of information in the literature about the association of MRONJ and Paget's disease. Even if osteonecrosis of the jaw could be a consequence of the disease, in this case, it is more in relation to the BP treatment.

Conclusions: Although MRONJ might be considered a rare condition in Paget's disease, patients prior to starting antiresorptive therapy and in particular iv BPs should have a complete dental examination and panoramic X-Ray.

Nemesis relevance: side effect of bisphosphonate treatment

Keywords Paget's disease, jaw osteonecrosis, bisphosphonates 
Introduction

Paget's disease of bone (PD) is characterized by a focal increase in bone resorption and accelerated bone formation leading to a weaker and disorganised bone. It can affect one or more site throughout the skeleton [1]. Described in 1876 by Sir James Paget, this "osteitis deformans" is the second most common metabolic bone disease after osteoporosis.

The disease affects preferentially a male population after $55 \mathrm{y}$. The incidence is higher in Western Europe, Australia and in America, affecting 2-7\% of the Caucasian population $[2,3]$.

The maxillofacial type occurs in less than $15 \%$ of patients, and the maxilla is more commonly affected than the mandible by a $2: 1$ ratio [4].

Treatment of PD tends to relieve bone pain and restore normal turnover. Bisphosphonates (BPs) have been the treatment of choice since the 1990s, either in the form of oral BPs (alendronate, risedronate...) or IV BPs (pamidronate, zoledronate). The frequency and dose of BPs treatment is determined by patient response [1].

Medication related osteonecrosis of jaws (MRONJ) was first described in 2003 under the name of bisphosphonate related osteonecrosis of the jaw $[5,6]$. The vast majority of cases occur in patients with advanced malignancies and skeletal metastases who have received frequent, high cumulative doses of antiresorptive therapy (IV BPs or denosumab). Less than 5\% of MRONJ occurs in non-cancer patients (osteoporosis, Paget's disease, fibrous dysplasia, rheumatoid arthritis...), which received lower and less frequent doses of oral, iv BPs or denosumab and presented with less comorbidity [3,7].

We report a case of Paget's disease of the skull and maxilla in a patient who received multiple courses of IV BPs and presented with 4 episodes of maxillary bone necrosis. 


\section{Case report}

An 87-year-old non-smoking Caucasian woman was referred to the outpatient maxillofacial department in 2009 for maxillary bone pain that had evolved over the previous 3 months. She was diagnosed with Paget's disease affecting the skull and maxilla in 1965 (Figure 1).

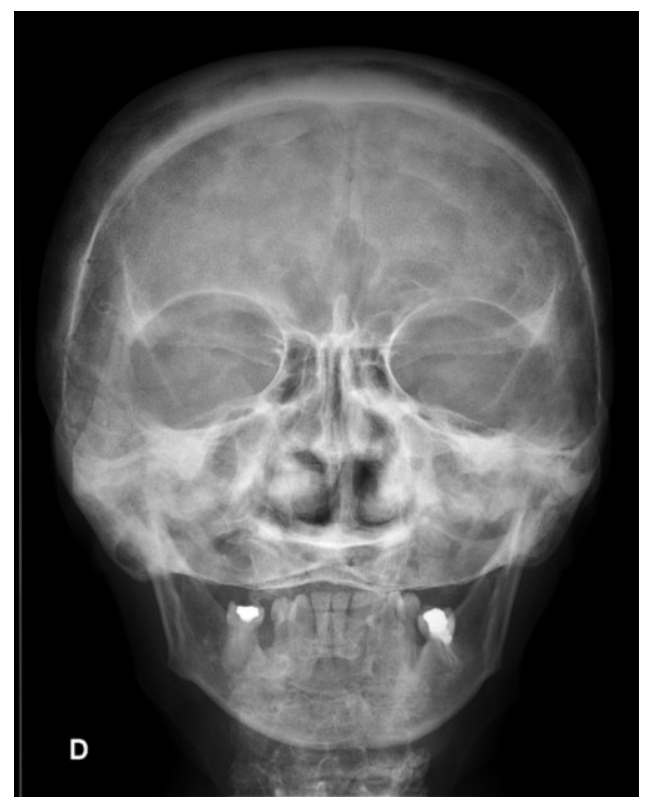

Fig. 1 Plain X-Ray demonstrating typical cotton wool aspect and bone enlargement in the maxillary and cranial vault.

No other comorbidity was ascertained. The patient received 11 infusions of pamidronate (30 to $60 \mathrm{mg}$ ) from 1993 to 1995 , with the last infusion occurring one month before she underwent maxillary surgical extractions and decortication to treat chronic infection and maxillary enlargement. At that time BPs were administrated to reduce the vascularity of bone to prevent excessive PD-related bleeding. Following these surgical extractions, the sockets failed to heal and bone exposure persisted despite numerous interventions. Two years after the extraction, there was still an area of bone exposure in the posterior left maxillary and a large sequestrum in the anterior right maxillary. After right sequestrectomy in 1997, she developed a right oroantral fistula. The left maxillary region was debrided and mucosa was closed. A 
maxillary obturator prosthesis was adjusted a few months later. BPs treatment was stopped for 5 years because the patient had not any symptoms from Paget's disease. During these five years, the patient was still free of intra-oral problems and bone exposure. Between 2002 and 2009, she received 6 courses of pamidronate $(180 \mathrm{mg}$ in 3 days) and 7 courses of IV alendronate ( $20 \mathrm{mg}$ in 2 days) for ocular problems (compression). In 2002, she sought treatment for left maxillary infection, probably due to compression of a poorly fitted prosthesis, which was complicated by bone exposure and a left maxillary sequestrum. She was treated with local debridement, sequestrectomy and wound closure. A larger oroantral fistula then developed on the treated left side. Her obturator prosthesis was adapted. In January 2009, one month after her last injection of alendronate, she presented with pain in the nasal spine region. On oral examination, bone exposure was detected in the left maxillary. A panoramic X-Ray (Figure 2), axial CT scan (Figure 3), coronal CT scan (Figure 4) and 3D CT scan (Figure 5) confirmed the presence of a left maxillary sequestrum. Spontaneous expulsion of the sequestrum $(2 \times 1 \mathrm{~cm}) 10$ weeks after the symptoms began revealed a normal underlying mucosa (Figure 6). Pathological examination found necrotic lamellar bone and bacteria colonies. The patient's serum alkaline phosphatase level, calcium and phosphate were within normal limits at that time. A new obturator prosthesis was made. The patient received a final course of IV alendronate in July 2009 and presented with a new episode of bone exposure and infection in March 2010 for which she received multiple course of antibiotics until resolution. 


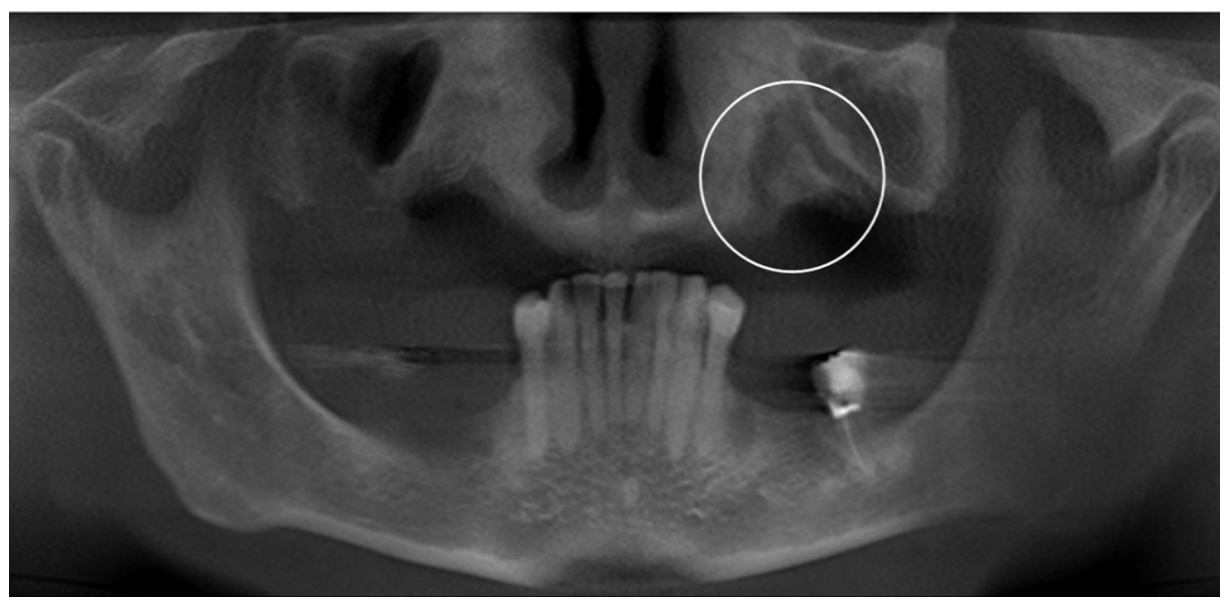

Fig. 2 Panoramic X-Ray, showing the same sequestrum and periostal reaction in the remaining maxillary bone. We can see the typical condensation of maxillary pagetic bone. Mandibular aspect seems to be normal.

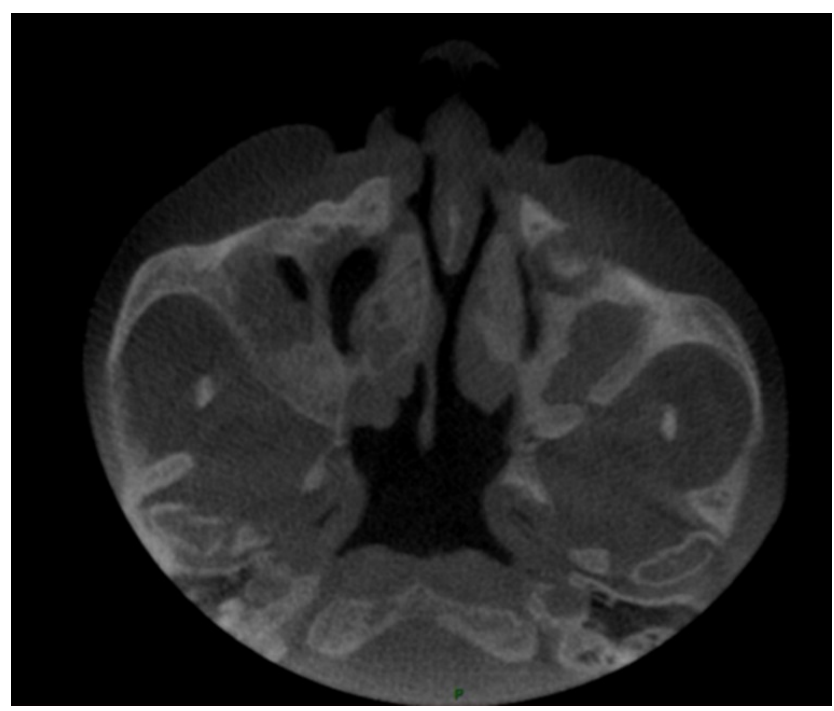

232

Fig. 3 CT scan demonstrating the left maxillary sequestrum in an axial view. 


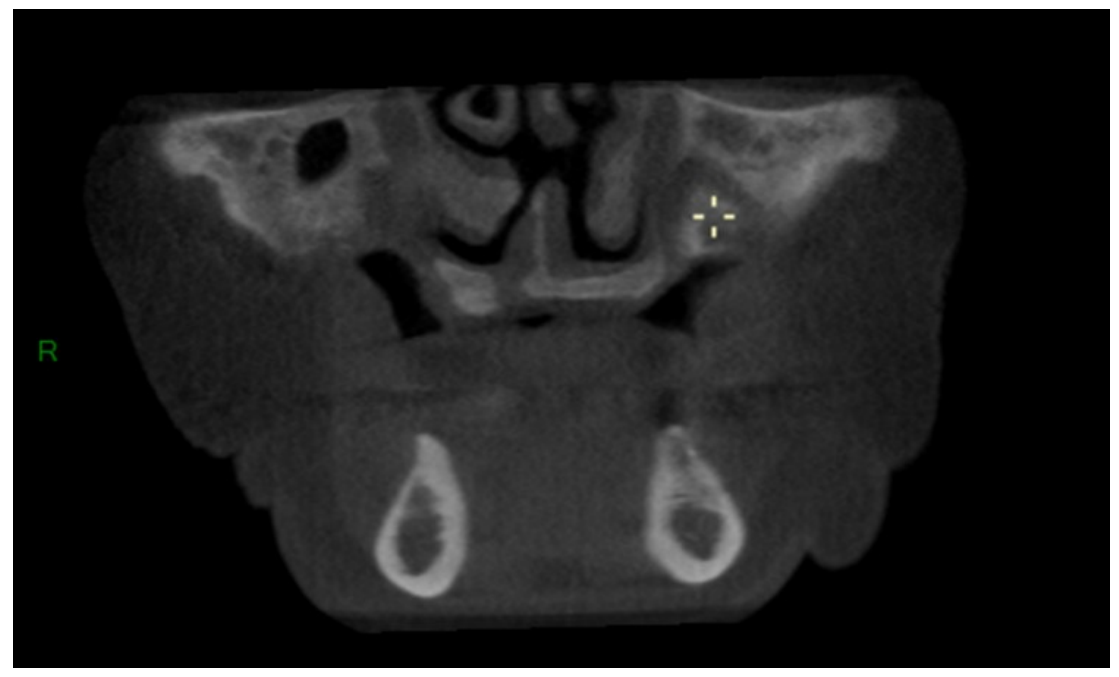

Fig. 4 CT scan demonstrating the left maxillary sequestrum in a coronal view

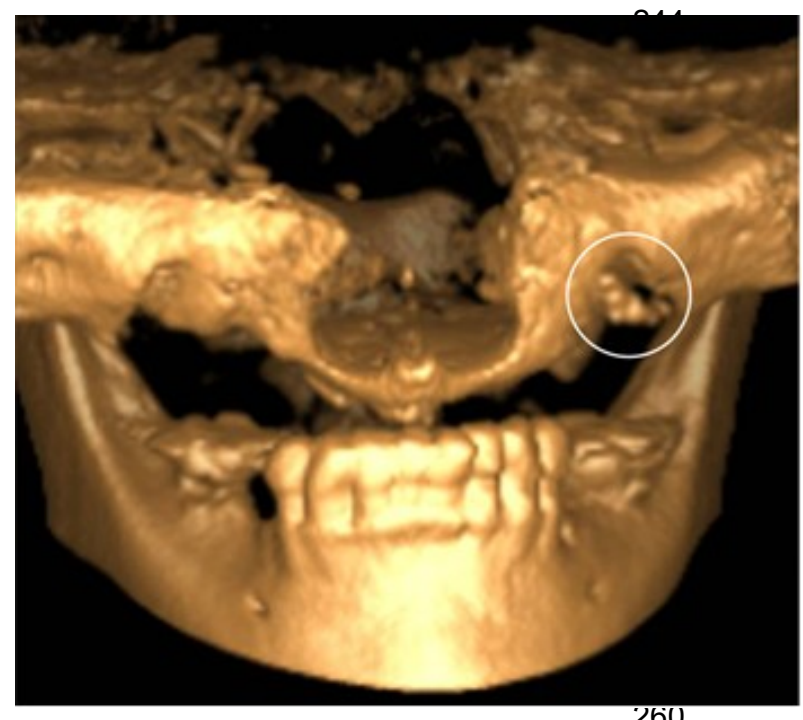

Fig. 5 3D maxillofacial CT scan showing sequestration in the left maxila. Enlargement of the maxillary is typical of Paget's disease. 


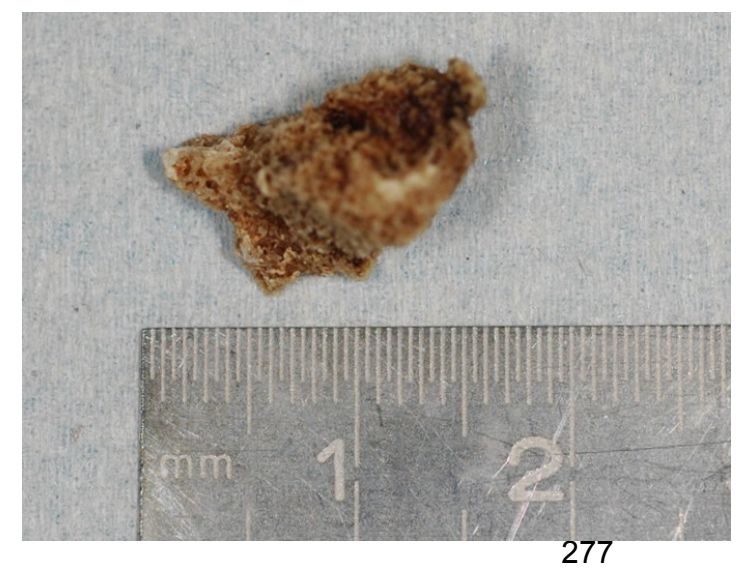

Fig. 6 Spontaneous expulsion of a bone sequester $2 \times 1 \mathrm{~cm}$

\section{Discussion}

Paget's disease (PD) of bone affects predominantly the skull, spine, hip, tibia and pelvis and can affect one or more site $[1,5]$. Maxillofacial involvement occurs in less than $15 \%$ of patients and maxilla is more commonly affected than mandible (ratio $2: 1$ ) [4].

Only $5 \%$ of patients with PD will develop symptoms including pain, skeletal deformity, fractures, compression according to the involved site, dental complications and rare sarcomatous degeneration $(<1 \%)$. When PD affects the jaw, the most common problems are associated with dental extraction due to hypercementosis and ankylosis, leading to surgical extractions. Complications are excessive bleeding in the vascular lytic phase and delayed healing and infection in the avascular phase [3]. Following extraction persistent sinuses may develop. Other dental complications include enlargement of the bone, migration of the teeth, malocclusion, loss of teeth, and osteomyelitis. Jaw bone sequestration is very uncommon, and is more often mentioned as a complication of BPs treatment than as a feature of PD of the maxilla [8].

Measurements of biological bone metabolism comprise parameters of bone formation such as serum alkaline phosphatase, C-terminal propeptide of type I collagen, $\mathrm{N}$-terminal propeptide of type I collagen as well as parameters of bone resorption such as serum or urinary $\mathrm{C}$-terminal telopeptide (CTX) and $\mathrm{N}$-terminal telopeptide (NTX) of type I collagen [2].

Monostotic PD of bone usually does not provoke significant elevation of serum alkaline phosphatase.

Aetiology of PD is unclear but genetic and viral components are suggested.

Mutations in the sequestome SQSTM1/p62 gene were identified in $46 \%$ of familial Paget cases and $16 \%$ of patients with sporadic PD. The presence of virus-like 
inclusions in the osteoclast nuclei, like paramyxovirus or syncitial respiratory virus has led to a viral hypothesis [1-3].

$\mathrm{PD}$ is considered to be a primary disorder of the osteoclast. The pathology is characterized by increased osteoclast bone resorption, followed by inadequate bone formation, leading to a disorganized bone with reduced mechanical strength [1]. Symptomatic PD is the main indication for treatment (pain, nerve compression...) [1].

Treatment aims at the suppression of osteoclast activity and is achieved with bisphosphonates.

The optimal regimen of BPs remains controversial. Oral formulations may be limited by complicated dosing regimens and poor gastrointestinal absorption. Currently, zoledronic acid is administrated as a single $5 \mathrm{mg}$ infusion and normalizes alkaline phosphatase in the majority of patients $[9,10]$. Denosumab had been used less frequently in patients refractory or intolerant to BPs [11].

Medication related osteonecrosis of the jaw is defined as an exposed bone or bone that can be probed through an intraoral or extraoral fistula in the maxillofacial region that has persisted for longer than 8 weeks in a patient with current or previous treatment with antiresorptive

or antiangiogenic agents, in the absence of radiation therapy to the jaws or obvious metastatic disease to the jaws [12].

The exact mechanism of MRONJ remains unclear. Alteration of bone turnover, hypovascularisation and infection seem to play a role. The majority of MRONJ are in relation with tooth extraction.

The real incidence of MRONJ is still unknown and varies between 1,2\% and 12,8\% in cancer patients. In a report of Mavrokokki [13], the overall incidence of MRONJ is 1 in 930 with 1 in 87 for cancer patient, 1 in 2260 for osteoporotic patients and 1 in 56 for Paget's disease. For other authors the overall maximum frequency of extraction-related MRONJ is 1 in 125, with 1 in 11 for bone metastasis, 1 in 296 for osteoporosis, and 1 in 7,4 for PD [14, 15].

Limited data are available about the risk of MRONJ in patients affected by non neoplastic diseases.

Only a few cases of MRONJ in PD patients have been reported in the literature. In a review in 2010, Filleul et al [16] analysed 2408 patients with MRONJ including $0,7 \%$ of patients with PD.

More recently, a systematic review from McGowan et al [17] identified 4106 patients with MRONJ. Twenty-four patients presented with non malignant systematic diseases other than osteoporosis $(0,5 \%)$ including 5 PD.

Even sequestration can be an exceptional but normal complication in PD, treatment with BPs may be the trigger factor of the osteonecrosis. In our patient, the first episode of bone necrosis appeared after oral surgery and repeated doses of iv BP. The 3 following episodes of bone exposure occurred each time after close BPs courses. The diagnosis of MRONJ was thus retained. We have no information about Paget's disease location in the literature reports. In our case report, maxillofacial 
location is probably a worsening factor because of accumulation of BPs at sites of active bone remodelling thus in the pagetic maxilla.

Although MRONJ might be considered a rare condition in Paget's disease, patients prior to starting antiresoptive therapy and in particular iv BPs should have a complete dental examination and panoramic X-Ray. If therapy can be delayed, oral infection should be treated, restorative care, dental extraction for non salvage teeth should be carried on. Use of soft liners on denture also seems prudent. Once BPs treatment is started, regular oral examination and preventive approach are important for early diagnosis and treatment if necessary. Patients should be informed about the risk of developing MRONJ in association with oral surgery and invasive dental procedures, even if dental implant placement. The poor quality of bone in PD renders it susceptible to infection. In these at risk patients, the only "intervention" with a proven decreased risk of MRONJ is prevention, as it is the case with radiotherapy.

This is the first description of a patient diagnosed with a maxillary localisation of Paget's disease of bone presenting 4 episodes of MRONJ in 15 years, each bone exposure following a course of iv BPs.

- Funding sources statement: authors received no funding for this case report

- Competing interests: all authors declare no competing interest

- Ethical approval: not applicable

- Informed consent: not applicable

\section{Authors contribution:}

\begin{tabular}{|l|l|}
\hline Author & Contributor role \\
\hline Magremanne M & $\begin{array}{l}\text { Conceptualization, Data curation, } \\
\text { Investigation, Methodology, Validation, } \\
\text { Resources, Writing original draft } \\
\text { preparation, Writing-review and editing }\end{array}$ \\
\hline Grisolle A & $\begin{array}{l}\text { Writing original draft preparation, Writing- } \\
\text { review and editing }\end{array}$ \\
\hline Ryechler H & Writing-review and editing \\
\hline
\end{tabular}

\section{References}

1. Wat WZ. Current perspectives on bisphosphonate treatment in Paget's disease of bone. Ther Clin Risk Manag 2014;10:977-983. 
2. Ralston SH, Langston AL. Pathogenesis and management of Paget's disease of bone. Lancet 2008;372:155-163.

3. Torres J, Tamimi F, Garcia I, Herrero A, Rivera B, Sobrino JA, Hernández G. Dental implants in a patient with Paget disease under bisphosphonate treatment: A case report. Oral Surg Oral Med Oral Pathol Oral Radiol Endod 2009; 107:387-392.

4. Lucas RB. The jaws and teeth in Paget's disease of bone. J Clin Path 1955;8:195-200.

5. Marx RE. Pamidronate (Aredia) and zoledronate (Zometa) induced avascular necrosis of the jaws: A growing epidemic. J Oral Maxillofac Surg 2003;61:1115-1117.

6. Ruggiero SL, Mehrotra B, Rosenberg TJ, Engroff SL. Osteonecrosis of the jaws associated with the use of bisphosphonates: A review of 63 cases. J Oral Maxillofac Surg 2004;62:527-534.

7. Shane E, Goldring S, Christakos S, Drezner M, Eisman J, Silverman S, Pendrys D. Osteonecrosis of the jaw: more research needed. J Bone Miner Res 2006;21:1503-1505.

8. Polisetti N, Neerupakam M, Prathi VS, Prakash J, Vaishnavi D, Beeraka SS, Bhavirisetty D. Osteonecrosis secondary to Paget's disease: radiological and pathological features. J Clin Imaging Sci 2014;4(Suppl 2):1.

9. Black DM, Delmas PD, Eastell R, Reid IR, Boonen S, Cauley JA, Cosman F, Lakatos P, Leung PC, Man Z, Mautalen C, Mesenbrink P, Hu H, Caminis J, Tong K, Rosario-Jansen T, Krasnow J, Hue TF, Sellmeyer D, Eriksen EF, Cummings SR; HORIZON Pivotal Fracture Trial. Once-yearly zoledronic acid for treatment of postmenopausal osteoporosis. N Engl J Med 2007;356:18091822.

10. Seton M, Krane SM. Use of zoledronic acid in the treatment of Paget's disease. Ther Clin Risk Manag 2007;3:913-918.

11. Reid IR, Sharma S, Kalluru R, Eagleton C. Treatment of Paget's disease of bone with Denosumab: Case report and literature review. Calcif Tissue Int 2016;99:322-325.

12. Ruggiero SL, Dodson TB, Fantasia J, Goodday R, Aghaloo T, Mehrotra B6, O'Ryan F; American Association of Oral and Maxillofacial Surgeons position paper on medication-related osteonecrosis of the jaw-2014 update. American Association of Oral and Maxillofacial Surgeons. J Oral Maxillofac Surg 2014;72:1938-1956. 
13. Mavrokokki T, Cheng A, Stein B, Goss A. Nature and frequency of bisphosphonate-associated osteonecrosis of the jaws in Australia. J Oral Maxillofac Surg 2007;65:415-423.

14. Assael LA. Oral bisphosphonates as a cause of bisphosphonate-related osteonecrosis of the jaws: clinical findings, assessment of risks, and preventive strategies. J Oral Maxillofac Surg 2009;67(suppl 1):35-43.

15. Lam DK, Sandor GK, Holmes HI, Evans AW, Clokie CM. A Review of bisphosphonate-associated osteonecrosis of the jaws and its management. J Can Dent Assoc 2007;73:417-422.

16. Filleul O, Crompot E, Saussez S. Bisphosphonate-induced osteonecrosis of the jaw: a review of 2,400 patient cases. J Cancer Res Clin Oncol 2010;136:11171124.

17. McGowan $\mathrm{K}$, McGowan $\mathrm{T}$, Ivanovski S. Risk factors for medication-related osteonecrosis of the jaws: A systematic review. Oral Dis. 2018;24:527-553. 